\title{
REGULAR EXTENSIONS AND THE SOLVABILITY OF OPERATOR EQUATIONS
}

\author{
ROGER H. HOMER ${ }^{1}$
}

Let $T_{1}$ be a closed linear operator in a complex Banach space. In this paper we are concerned with the effect of changes in the complex parameter $\lambda$ on the solvability of the equation $\left(T_{1}-\lambda I\right) x=y$. By introducing the notion of a regular extension we are able to generalize a result of A. S. Markus [2].

In order to introduce extension terminology we consider $T_{1}$ to be an extension of a closed linear operator $T_{0}$, denoted $T_{0} \subset T_{1}$. We use $D(T)$ to denote the domain of an operator $T, R(T)$ for the range of $T$ and $N(T)$ for the null space of $T . K(T)$ denotes the set of all elements $y$ such that the equation $T^{n} x=y$ is solvable for every positive integer $n$, a concept originally introduced by $\mathrm{F}$. Riesz [3, p. 87].

We call a closed linear operator $T$ a regular extension at $\lambda$ if $T_{0} \subset T \subset T_{1}, R(T-\lambda I)_{-}=R\left(T_{1}-\lambda I\right)$ and $T-\lambda I$ has a bounded inverse. We call $T$ a regular extension near $\lambda_{0}$ if for every $\lambda$ in some neighborhood of $\lambda_{0}, T$ is a regular extension at $\lambda$. Let $\Gamma$ be any connected component of the open set consisting of all complex numbers $\lambda$ such that there exists a regular extension near $\lambda$.

Theorem. For each element $y$, either $y$ belongs to $K\left(T_{1}-\lambda I\right)$ for all $\lambda$ in $\Gamma$, or there is no $\lambda$ in $\Gamma$ such that $y$ belongs to $K\left(T_{1}-\lambda I\right)$ and the set of all $\lambda$ in $\Gamma$ such that $y$ belongs to $R\left(T_{1}-\lambda I\right)$ has no accumulation point in $\Gamma$.

The above mentioned result of A. S. Markus [2] differs from this only in the hypothesis. He takes $\Gamma$ to be an open connected set such that for every $\lambda$ in $\Gamma, R\left(T_{1}-\lambda I\right)$ is closed and $N\left(T_{1}-\lambda I\right)$ is of finite dimension independent of $\lambda .{ }^{2}$ Our hypothesis implies that $R\left(T_{1}-\lambda I\right)$ is closed for $\lambda$ in $\Gamma$, but admits certain instances of $N\left(T_{1}-\lambda I\right)$ being infinite dimensional.

We find it convenient to employ several lemmas in the proof of the theorem. The idea for Lemma 1 came from a paper by I. C. Gokhberg

Received by the editors July 23, 1960.

1 The material in this paper was included in the author's Doctoral Dissertation submitted to the University of California, Berkeley under the guidance of Professor Frantisek Wolf. The research was supported, in part, by NSF Grant G-5010.

2 It is shown in the author's dissertation that if $R\left(T_{1}-\lambda_{0} I\right)$ is closed and $N\left(T_{1}-\lambda_{0} I\right)$ is finite dimensional then there exists a regular extension at $\lambda_{0}$. Such an extension is shown to be regular near $\lambda_{0}$ if and only if the dimension of $N\left(T_{1}-\lambda I\right)$ is constant in some neighborhood of $\lambda_{0}$. 
and A. S. Markus [1] dealing with an operator at points of regular type.

Lemma 1. Let $T$ be a regular extension near $\lambda_{0}$ and $\left\{\lambda_{j}\right\}$ be a sequence such that $\lim _{j \rightarrow \infty} \lambda_{j}=\lambda_{0}$ and $\lambda_{j} \neq \lambda_{0}$ for each $j$. If $y$ is in $R\left(T_{1}-\lambda_{j} I\right)$ for every $j$ then $y$ is in $K\left(T-\lambda_{0} I\right)$.

Proof. Because of linearity we may assume without loss of generality that $\lambda_{0}=0$. By the definitions of regular extensions there exists a neighborhood of 0 such that for each $\lambda$ in this neighborhood $(T-\lambda I)^{-1}$ exists as a bounded operator defined on $R\left(T_{1}-\lambda I\right)$. By possibly omitting a finite number of terms from $\left\{\lambda_{j}\right\}$ we may assume that $\left\{\lambda_{j}\right\}$ is contained in such a neighborhood. Thus $y$ belongs to $R\left(T-\lambda_{j} I\right)$ for every $j$.

For each $j$ let $u_{j}$ be a solution of $\left(T-\lambda_{j} I\right) x=y$. Then $T u_{j}=\lambda_{j} u_{j}+y$ and since $T^{-1}$ is bounded,

$$
\begin{aligned}
\left\|u_{j}\right\| & \leqq\left\|T^{-1}\right\| \cdot\left\|\lambda_{j} u_{j}+y\right\| \\
& \leqq\left\|T^{-1}\right\| \cdot\left|\lambda_{j}\right| \cdot\left\|u_{j}\right\|+\left\|T^{-1}\right\| \cdot\|y\| .
\end{aligned}
$$

Thus for sufficiently large $j$ we have

$$
\left\|u_{j}\right\| \leqq \frac{\left\|T^{-1}\right\| \cdot\|y\|}{1-\left|\lambda_{j}\right| \cdot\left\|T^{-1}\right\|} \leqq 2\left\|T^{-1}\right\| \cdot\|y\|,
$$

and $\left\{u_{j}\right\}$ is a bounded sequence.

Now $\lim _{j \rightarrow \infty} T u_{j}=y$ so $y$ belongs to $R(T)$ which is closed since it is the domain of the closed, bounded operator $T^{-1}$. Let $x_{0}$ be the solution of $T x=y$. Notice that $\left(1 / \lambda_{j}\right)\left(u_{j}-x_{0}\right)=T^{-1} u_{j}$ and because $\left\{\left\|T^{-1} u_{j}\right\|\right\}$ is bounded we have $\lim _{j \rightarrow \infty} u_{j}=x_{0}$. Assume the induction hypothesis that $x_{0}, x_{1}, \cdots, x_{n}$ satisfy the following conditions: $T x_{0}=y, T x_{k}$ $=x_{k-1}(k=1,2, \cdots, n)$ and $\lim _{j \rightarrow \infty} w_{j, n}=x_{n}$ where

$$
w_{j, n}=\left(\frac{1}{\lambda_{j}}\right)^{n}\left[u_{j}-\sum_{k=0}^{n-1}\left(\lambda_{j}\right)^{k} x_{k}\right] \text {. }
$$

Then $\lim _{j \rightarrow \infty} w_{j, n+1}$ exists since $\left\{w_{j, n}\right\}$ converges, $T^{-1}$ is bounded and $T w_{j, n+1}=w_{j, n}$. Let $x_{n+1}=\lim _{j \rightarrow \infty} w_{j, n+1}$ and observe $T x_{n+1}=x_{n}$ because $T$ is closed. Thus by induction $y$ belongs to $K(T)$.

LEMMA 2. If $T$ is a regular extension near $\lambda_{0}$ then $N\left(T_{1}-\lambda_{0} I\right)$ is contained in $K\left(T-\lambda_{0} I\right)$.

Proof. Again assume $\lambda_{0}=0$ and $\left\{\lambda_{j}\right\}$ is a sequence such as in Lemma 1. Suppose $T_{1} x=0$. Then $\left(T_{1}-\lambda_{j} I\right)\left(x /-\lambda_{j}\right)=x$ and $x$ belongs to $K(T)$ by Lemma 1 . 
Lemma 3. If $T$ is a regular extension near $\lambda, m$ is a non-negative integer and $x \in D\left(\left(T_{1}-\lambda I\right)^{m+1}\right)$ then $(T-\lambda I)^{-1}\left(T_{1}-\lambda I\right)^{m+1} x$ $\in R\left(\left(T_{1}-\lambda I\right)^{m}\right)$.

Proof. Let $\lambda=0, S=T^{-1}, x \in D\left(T_{1}^{m+1}\right)$ and $z=T_{1}^{m} x-S T_{1}^{m+1} x$. Then $T_{1} z=T_{1}^{m+1} x-T_{1} S T_{1}^{m+1} x=0$ so by Lemma $2, z \in K(T)$ which is contained in $K\left(T_{1}\right)$. Let $v$ be such that $T_{1}^{m} v=z$ and observe $T_{1}^{m}(x-v)$ $=T_{1}^{m} x-T_{1}^{m} x+S T_{1}^{m+1} x=S T_{1}^{m+1} x$.

Lemma 4. If $T$ is a regular extension near $\lambda, S=(T-\lambda I)^{-1}$ and $y \in K\left(T_{1}-\lambda I\right)$ then for each non-negative integer $n, S^{n} y$ is defined and belongs to $K\left(T_{1}-\lambda I\right)$.

Proof. Let $\lambda=0$ and $y \in K\left(T_{1}\right)$. Then $S^{0} y=y \in K\left(T_{1}\right)$. Assume the induction hypothesis that $S^{n} y \in K\left(T_{1}\right)$. Since $K\left(T_{1}\right)$ is contained in $R\left(T_{1}\right)=D(S)$ we see that $S^{n+1} y$ is defined. Consider the equation $T_{1}^{m} x=S^{n+1} y$. By the induction hypothesis there is a $u$ such that $T_{1}^{m+1} u=S^{n} y$ and then by Lemma 3 there is a $v$ such that $T_{1}^{m} v=S T_{1}^{m+1} u$. Thus $S^{n+1} y=S S^{n} y=S T_{1}^{m+1} u=T_{1}^{m} v$ and since $m$ is arbitrary we have $S^{n+1} y \in K\left(T_{1}\right)$.

Note that this shows $K(T-\lambda I)=K\left(T_{1}-\lambda I\right)$.

Lemma 5. Let $T$ be a regular extension near $\lambda_{0}$ and $S=\left(T-\lambda_{0} I\right)^{-1}$. Then $y$ belongs to $R\left(T_{1}-\lambda I\right)$ for $y$ in $K\left(T_{1}-\lambda_{0} I\right)$ and for all $\lambda$ such that $\left|\lambda-\lambda_{0}\right| \cdot\|S\|<1$.

Proof. Let $\lambda_{0}=0, y \in K\left(T_{1}\right)$ and $\lambda$ satisfy $|\lambda| \cdot\|S\|<1$. By Lemma $4, S^{n} y$ is defined for all $n$ and $\sum_{j=0}^{\infty}(\lambda S)^{i} y$ is convergent because $\|\lambda S\|<1$. Since $S$ has a closed domain we see that $u=\sum_{j=0}^{\infty}(\lambda S)^{i} y$ $\in D(S)$. Finally $x=S u$ satisfies

$$
\begin{aligned}
\left(T_{1}-\lambda I\right) x & =\left(T_{1}-\lambda I\right) S \sum_{j=0}^{\infty}(\lambda S)^{j} y \\
& =T_{1} S y+T_{1} S \sum_{j=1}^{\infty}(\lambda S)^{j} y-\lambda S \sum_{j=0}^{\infty}(\lambda S)^{i} y \\
& =y+\sum_{j=1}^{\infty}(\lambda S)^{j} y-\sum_{j=0}^{\infty}(\lambda S)^{i+1} y=y .
\end{aligned}
$$

Lemma 6. $K\left(T_{1}-\lambda I\right)$ is independent of $\lambda$ in $\Gamma$.

Proof. For each element $y$ let $\Gamma_{y}$ be the set of all $\lambda \in \Gamma$ such that $y \in K\left(T_{1}-\lambda I\right)$. We will show that $\Gamma_{y}$ is both closed and open in $\Gamma$ and hence either void or all of $\Gamma$ since $\Gamma$ is connected.

If $\lambda_{0} \in \Gamma \cap \Gamma_{y}$ then $\lambda_{0} \in \Gamma_{y}$ by Lemma 1 . Hence $\Gamma_{y}$ is closed in $\Gamma$. 
If $\lambda_{0} \in \Gamma_{y}$ then Lemma 5 implies the existence of a neighborhood of $\lambda_{0}$ which is contained in $\Gamma$ and such that $y \in R\left(T_{1}-\lambda I\right)$ for every $\lambda$ in this neighborhood. For each such $\lambda$ we see that $\lambda \in \Gamma_{y}$ by Lemma 1 . Thus $\Gamma_{y}$ is open in $\Gamma$.

With Lemma 6 , the following observation completes the proof of the theorem.

Suppose $\lambda_{0} \in \Gamma$ is an accumulation point of the set of all $\lambda$ such that $y \in R\left(T_{1}-\lambda I\right)$. By Lemma $1, y \in K\left(T_{1}-\lambda_{0} I\right)$ since $K\left(T_{1}-\lambda_{0} I\right)$ $=K\left(T-\lambda_{0} I\right)$ if $T$ is a regular extension near $\lambda_{0}$.

\section{REFERENCES}

1. I. C. Gokhberg and A. S. Markus, On one characteristic property of the kernel of a linear operator, Dokl. Akad. Nauk SSSR vol. 105 (1955) pp. 893-896 (in Russian).

2. A. S. Markus, On a characteristic property of the kernel of a linear operator, Dokl. Akad. Nauk SSSR vol. 105 (1955) pp. 1144-1146 (in Russian).

3. F. Riesz, Über lineare Funktionalgleichungen, Acta Math. vol. 41 (1918) pp. 71-98.

University of California, Berkeley and

IOWA State University 